\title{
Acute reconstruction results in less sick-leave days and as such fewer indirect costs to the individual and society compared to delayed reconstruction for $A C L$ injuries
}

\author{
Christoffer von Essen $^{1}\left[\right.$ - Sebastian McCallum ${ }^{1} \cdot$ Björn Barenius $^{1} \cdot$ Karl Eriksson $^{1}$
}

Received: 14 December 2018 / Accepted: 1 February 2019 / Published online: 14 February 2019

(c) The Author(s) 2019

\begin{abstract}
Purpose To compare the total number of sick-leave days caused by the knee injury from the day of injury and over the first year between acute (within 8 days) and delayed (6-10 weeks) anterior cruciate ligament reconstruction (ACLR) and also assess other clinical outcomes during this period.

Methods Seventy patients with an acute ACL injury and Tegner level of 6 or more were randomized to acute (within 8 days) or delayed (after 6-10 weeks) ACLR. Patient-reported outcomes; objective IKDC and manual stability measurements were assessed at 6 and 12 months. With data from the Swedish Social Insurance Agency (Försäkringskassan) information about the number of sick-leave days due to the knee injury over the following 12 months was collected and compared between the two groups.

Results Seventy-one percent received compensation for sick leave (26 in the acute versus 23 in the delayed group). The mean number of sick-leave days for the acute group was significantly lower $(M=56.9, \mathrm{SD}=36.4)$ compared to the delayed group $(M=88.5, \mathrm{SD}=50.2), p<0.05$. The acute group was also significantly stronger in flexion in both slow and fast angle velocities according to Biodex ${ }^{\circledR}$. No other differences were found between the groups in other clinical assessments or in terms of associated injuries.

Conclusion Acute and delayed ACLR provided comparable clinical outcomes after 12 months. Acute reconstruction resulted in less sick-leave days and as such fewer indirect costs to the individual and society. These findings suggest that if patients requiring ACLR can be identified early and ACLR can be performed in the acute phase, socioeconomic costs can potentially be reduced by minimizing time off work.
\end{abstract}

Level of evidence II.

Keywords ACL $\cdot$ Acute $\cdot$ Outcome $\cdot$ Range of motion $\cdot$ Reconstruction $\cdot$ Costs

\section{Introduction}

A rupture of the anterior cruciate ligament (ACL) is a serious knee injury most commonly affecting young, physically active persons. In both male and female athletes, ACL injury is the most frequent cause of permanent disability [1]. The incidence of ACL injury is around 7000 cases per year in Sweden with approximately 3500 ACL reconstructions (ACLR) performed annually [2].

Christoffer von Essen

Christoffer.vonessen@gmail.com

1 Department of Orthopaedics, Stockholm South Hospital, Karolinska Institutet, Stockholm, Sweden
ACL injuries may be managed non-operatively with physical therapy and activity modification or with surgical reconstruction of the ligament. Although conservative treatment can be successful in some patient groups [3], it has been proven less successful in physically active patients who compete or train at a high level [4-6].

The optimal timing of surgery has been debated [7-10], with surgical reconstruction traditionally delayed for at least 6 weeks due to evidence that earlier reconstruction may increase the risk of developing arthrofibrosis [11-13]. However, recent studies have demonstrated that with newer surgical techniques, surgery may be performed in the acute phase following the injury without significantly increasing the risk of complications [8-10]. 
An ACL injury is functionally disabling and may predispose the knee to further injuries, such as chondral and meniscal injury, while also contributing to early onset of degenerative changes in the knee [1, 14-18]. In addition to potentially reducing these risks, surgery in the acute phase following injury may facilitate earlier return to work, resulting in socioeconomic benefits to both society and the individual. There is currently conflicting evidence regarding the socioeconomic costs of acute versus delayed surgery, with two recent studies finding early ACLR to be more costeffective than delayed surgery $[19,20]$ and another finding the opposite to be true [21].

In Sweden patients are entitled to paid sick leave if a doctor determines that work is not possible due to illness or injury, with up to $80 \%$ of income reimbursed. Costs are covered by the patient's employer during the first 2 weeks of leave and subsequently by the government.

The primary aim of this study was to compare the total number of sick-leave days taken following ACL rupture in those undergoing acute and delayed reconstruction, as a means of measuring socioeconomic cost. The secondary aim was to compare early functional outcomes between the groups. It was hypothesized that an acute ACLR results in lesser sick-leave days without inferior patient-reported outcomes.

\section{Materials and methods}

From 2006 to 2013, 2088 patients who had presented to the emergency department with an acute knee injury were followed up within 3 days at a knee clinic. Clinical examination and magnetic resonance imaging (MRI) were performed. If an ACL rupture was diagnosed and the patient consented to participation, they were assessed for inclusion in the study. The inclusion criteria for the study were: unilateral primary ACL injury in patients between 18 and 40 years of age with no previous knee injury to either leg, Tegner activity level [22] minimum 6, no additional meniscus or cartilage damage on MRI indicating the need for major acute meniscus or cartilage surgery, logistic availability to reconstruct the patient within 8 days of injury, no MCL-injury greater than grade 1 and no LCL-injury in need of surgery.

If all the pre-requisites were fulfilled, a research nurse performed randomization with the sealed envelope technique in the same session. Patient demographics are presented in Table 1 . The patients were prospectively randomized to reconstruction of the ACL either within 8 days from injury or with delayed reconstruction after recovery of range of motion (ROM), within 6-10 weeks from injury. The patients randomized to delayed surgery received pre-operative physiotherapy to restore normal range of movement and to preserve muscle strength.
At the time of inclusion and randomization the patients were evaluated regarding ROM (passive ROM measured with a goniometer and reported as a deficit in extension and flexion), instrumented laxity using the Rolimeter and thigh circumference measured $10 \mathrm{~cm}$ proximal to the proximal pole of the patella. Subjective and self-assessed IKDC [23], KOOS [24], Lysholm [22] and Tegner activity level were also evaluated.

In all clinical tests, the contralateral non-injured side was used as a reference.

\section{Surgical method}

All patients underwent an arthroscopic ACLR with a hamstring tendon autograft. If the single semitendinosus tendon was not sufficient in length or thickness, the gracilis tendon was also harvested. In the beginning of the study the tibia was drilled first and transtibial drilling of the femur was used. The tibia angle was $45^{\circ}-50^{\circ}$ depending on the surgeon's preferences, and a femur entry-point at 10 or 2 o'clock was preferred. Later in the study, due to the evolution of surgical technique at the time, the method for tunnel placement was changed. Drilling of the femoral tunnel was done through the anteromedial portal and the aim was to place the tunnel in the centre of the native footprint. The graft was fixed in the femur with an Endobutton continuous loop ${ }^{\circledR}$ (Smith \& Nephew, Inc., Andover, MA 01810, USA). In the tibia the graft was fixed with a metal interference-screw, RCI ${ }^{\circledR}$ (Smith \& Nephew, Inc., Andover, MA 01810, USA) or Soft Screw ${ }^{\circledR}$ (Arthrex Inc., Naples, Florida 34108, USA). As an additional reinforcement the graft was fixed with an osteo-suture over a "bone-bridge" in the tibia. Injured menisci were sutured if an injury was discovered during the surgery.

\section{Rehabilitation}

The rehabilitation was standardized to one physiotherapy centre and the same rehabilitation protocol was used for all patients. Closed-chain exercises and range of motion training was initiated within 1 week after the surgery. Open chain exercises were allowed after 6 weeks, running allowed after 14 weeks and resumption of sport activity after Biodex ${ }^{\circledR}$ testing showed $90 \%$ strength in injured leg compared to the contralateral leg, but never earlier than 6 months.

\section{Postoperative follow-up}

At 3 months ROM and circumference of the thighs were assessed by the patient's physiotherapist. An independent physiotherapist not involved in the rehabilitation assessed the patients at 6 and 12 months. 
Table 1 Descriptives of the study population

\begin{tabular}{|c|c|c|c|c|}
\hline & Total $(n=69)$ & Acute ACLR $(n=34)$ & $\begin{array}{l}\text { Delayed } \\
\text { ACLR }(n=35)\end{array}$ & Sign \\
\hline Age at inclusion mean $\pm \mathrm{SD}$ & $26.9 \pm 6.1$ & $27.7 \pm 6.5$ & $26.1 \pm 5.7$ & n.s. \\
\hline Gender: females $n(\%)$ & $21(31)$ & $10(30)$ & $11(31)$ & n.s. \\
\hline Height $\mathrm{cm}$ mean $\pm \mathrm{SD}$ & $177 \pm 9$ & $177 \pm 9$ & $178 \pm 9$ & n.s. \\
\hline Weight kg mean \pm SD & $77 \pm 11$ & $76 \pm 11$ & $78 \pm 12$ & n.s. \\
\hline Smoker $n(\%)$ & $4(6)$ & $2(6)$ & $2(6)$ & n.s. \\
\hline Highest education $(n=64)(\%)$ & & & & n.s. \\
\hline High school/college & $35(55)$ & $20(65)$ & $15(45)$ & \\
\hline University & $29(45)$ & $11(35)$ & $18(54)$ & \\
\hline Main occupation $n(\%)$ & & & & n.s. \\
\hline Working & $52(75)$ & $27(79)$ & $25(71)$ & \\
\hline Heavy manual labour & $12(23)$ & $8(30)$ & $4(16)$ & \\
\hline Light manual labor & $20(38)$ & $10(37)$ & $10(40)$ & \\
\hline Office work & $17(33)$ & $8(30)$ & $9(36)$ & \\
\hline No compensation & $3(6)$ & $1(4)$ & $2(8)$ & \\
\hline Student & $17(25)$ & $7(21)$ & $10(29)$ & \\
\hline Type of activity when injured $n(\%)$ & & & & n.s. \\
\hline Soccer & $27(38)$ & $14(41)$ & $13(37)$ & \\
\hline Indoor floorball & $16(24)$ & $6(18)$ & $10(29)$ & \\
\hline Alpine ski/snowboard & $10(15)$ & $7(20)$ & $3(8)$ & \\
\hline Handball & $5(7)$ & $1(3)$ & $4(11)$ & \\
\hline Wrestling/martial arts & $3(5)$ & $3(9)$ & 0 & \\
\hline Gymnastics & $2(3)$ & $2(6)$ & 0 & \\
\hline Ice hockey & $1(2)$ & 0 & $1(3)$ & \\
\hline Am. football & $1(2)$ & 0 & $1(3)$ & \\
\hline Badminton & $1(2)$ & 0 & $1(3)$ & \\
\hline Basketball & $1(2)$ & 0 & $1(3)$ & \\
\hline Dance & $1(2)$ & $1(3)$ & 0 & \\
\hline Tennis & $1(2)$ & 0 & $1(3)$ & \\
\hline
\end{tabular}

Patient demographics at baseline are displayed as mean \pm SD, number and percentage, respectively
The follow-up at 6 and 12 months included the same subjective scores as pre-operatively as well as ROM, circumference of the thighs and single leg hop. Isokinetic peak torque strength at $60 \% \mathrm{~s}, 180 \% \mathrm{~s}$ and $240 \%$ s, and isometric torque strength at $60^{\circ}$ and $180^{\circ}$, in both extension and flexion was measured with Biode ${ }^{\circledR}[25]$.

The number of sick-leave days claimed by each patient within the first 12 months from the injury was obtained from the government agency responsible for sick-leave payments (Swedish Social Insurance Agency-SSIA, http://www.forsakringskassan.se). Only sick-leave days based on a diagnosis of ACL injury were included.

The study was approved by the regional ethics committee at the Karolinska Institute, Stockholm Sweden (reference no. 2006/404-31/3/2008/1541-32).

\section{Statistical analysis}

Statistical analysis was performed with the IBM SPSS 22.0 software package for Macintosh. Nominal variables were tested by the $\chi^{2}$ test or the Fisher exact test. Ordinal variables and non-normality distributed interval and ratio scale variables were evaluated by the Mann-Whitney $U$ test. Student's $t$ test was used for normally distributed interval and ratio scale variables in independent groups. Longitudinal statistics were done with the paired-samples $t$ test for normally distributed scale variables and the Wilcoxon signed rank test for ordinal and non-normality distributed scale variables. The tests were 2 -sided. The results were considered significant at $p<0.05$.

The study was originally designed to compare range of motion in the knee; hence students and patients who did not 
register for sick leave were not excluded. Furthermore, no power analysis regarding differences in days of sick leave was performed prior to study commencement, a post hoc power analysis using $\mathrm{G}^{*}$ Power 3.1.9.2 (Franz Paul, Kiel, Germany) was used to determine the power of the present study. Based on number and the distribution of sick-leave days at 1 year in the study population, an effect size of 0.73 was calculated, with $\alpha$ set at 0.05 and 2 -sided tests, the power of the study was calculated to $71 \%$.

\section{Results}

Seventy patients were included, with 35 patients randomized to early ACLR and 35 patients to late reconstruction. One patient from the delayed group dropped out before surgery due to personal reasons, a second was excluded as they were unable to participate in follow-up according to the study protocol (Fig. 1).

Demographic data of the study groups are displayed in Table 2. At the 12-month follow-up, 66 (94\%) patients were available for the clinical follow-up. Three from the acute and one from the delayed group was missing regarding clinical results. The only significant difference between the groups at the time of surgery was the time between injury and reconstruction.
Table 2 Demographics

\begin{tabular}{llll}
\hline & $\begin{array}{l}\text { Acute } \\
\text { ACLR } \\
(n=34)\end{array}$ & $\begin{array}{l}\text { Delayed } \\
\text { ACLR } \\
(n=35)\end{array}$ & Sign. \\
\hline Time injury-recon $(\mathrm{d} \pm \mathrm{SD})$ & $5 \pm 2$ & $55 \pm 8$ & $<0.01$ \\
OP time $(\min \pm \mathrm{SD})$ & $93 \pm 20$ & $83 \pm 18$ & n.s. \\
Graft diameter $(\mathrm{mm} \pm \mathrm{SD})$ & $8.8 \pm 0.8$ & $8.6 \pm 0.8$ & n.s. \\
Additional injury $[n(\%)]$ & $21(66)$ & $15(47)$ & n.s. \\
Medial meniscus $[n(\%)]$ & $7(21)$ & $2(6)$ & n.s. \\
Lateral meniscus $[n(\%)]$ & $13(39)$ & $10(29)$ & n.s. \\
Sutures $[n(\%)]$ & $3(9)$ & $1(3)$ & n.s. \\
Cartilage inj. $[n(\%)]$ & $10(29)$ & $4(11)$ & n.s. \\
Transtibial technique $[n(\%)]$ & $12(35)$ & $10(29)$ & n.s. \\
Anteromedial technique $[n(\%)]$ & $22(65)$ & $25(71)$ & n.s. \\
\hline
\end{tabular}

Patient demographics at baseline for patients who underwent ACLR are displayed as mean $\pm \mathrm{SD}$, number and percentage, respectively. Statistically significant $(p<0.05)$ values were only seen for the time from injury to reconstruction

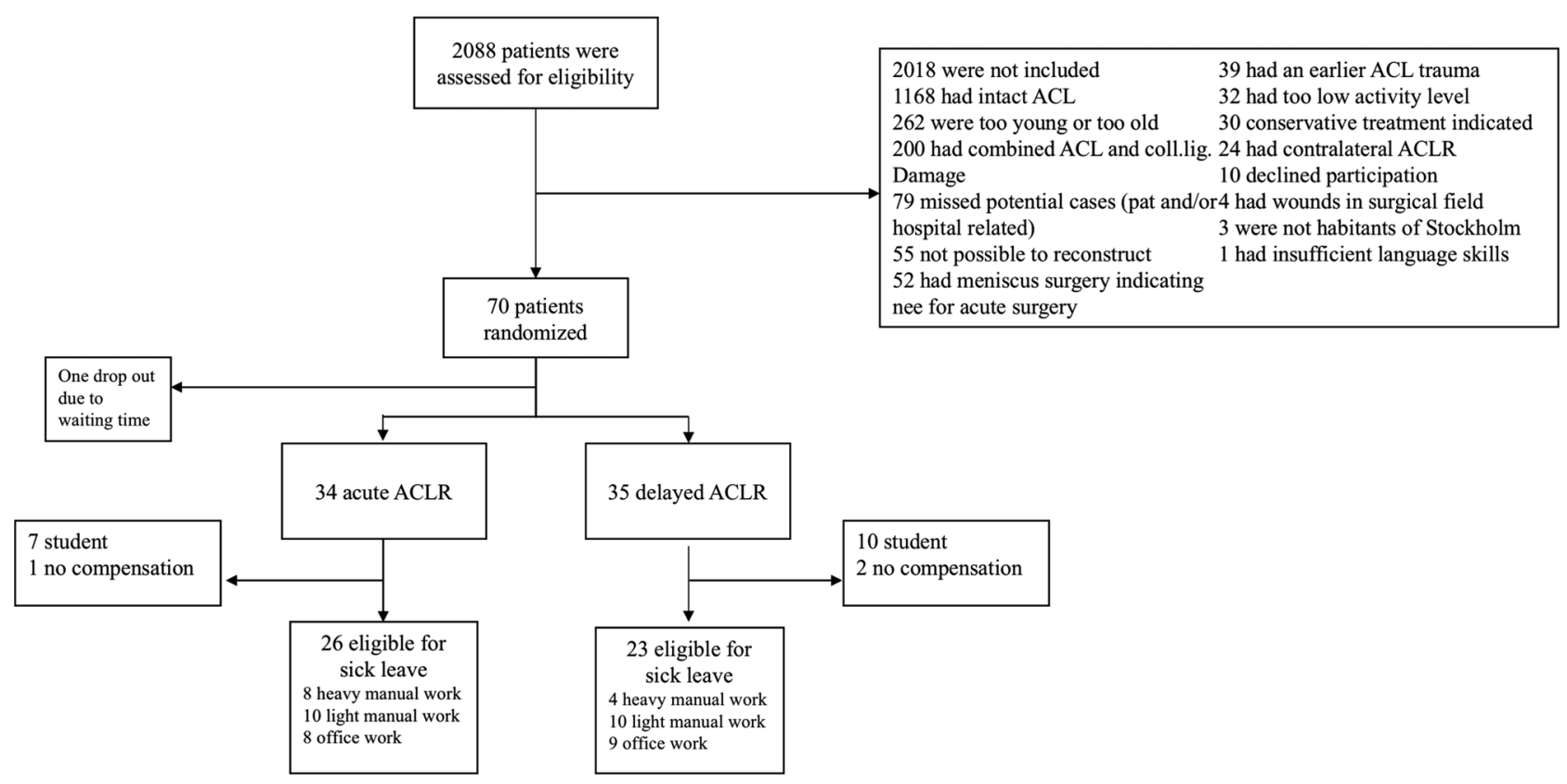

Fig. 1 Enrollment, randomization and sick-leave compensation of subjects 


\section{Sick-leave days}

All patients were assessed regarding sick leave during the first year. Seventeen (25\%) patients out of 69 were students without registered compensation for sick leave. An additional 3 (4\%) patients were not students, but were not registered for any compensation for sick leave from work; these patients were predominantly self-employed and did not claim any sick leave compensation. 49 out of $69(71 \%)$ patients received compensation for sick leave, 26 in the acute group and 23 in the delayed group. No differences were seen in regard to occupations involving heavy or light manual labor; Table 1.

Total amount of days' sick leave by study group are presented in Fig. 2. The mean number of sick-leave days for the acute group was significantly lower $(M=56.9, \mathrm{SD}=36.4)$ compared to the delayed group $(M=88.5, \mathrm{SD}=50.2)$, $p<0.05$.

Distribution of sick-leave days differed between the groups, the acute group had one continuous period, while the delayed had either one continuous or two separate periods depending on the physical demands of the occupation. The delayed group was on sick leave for a mean of 23 days before reconstruction.

Total amount sick-leave days

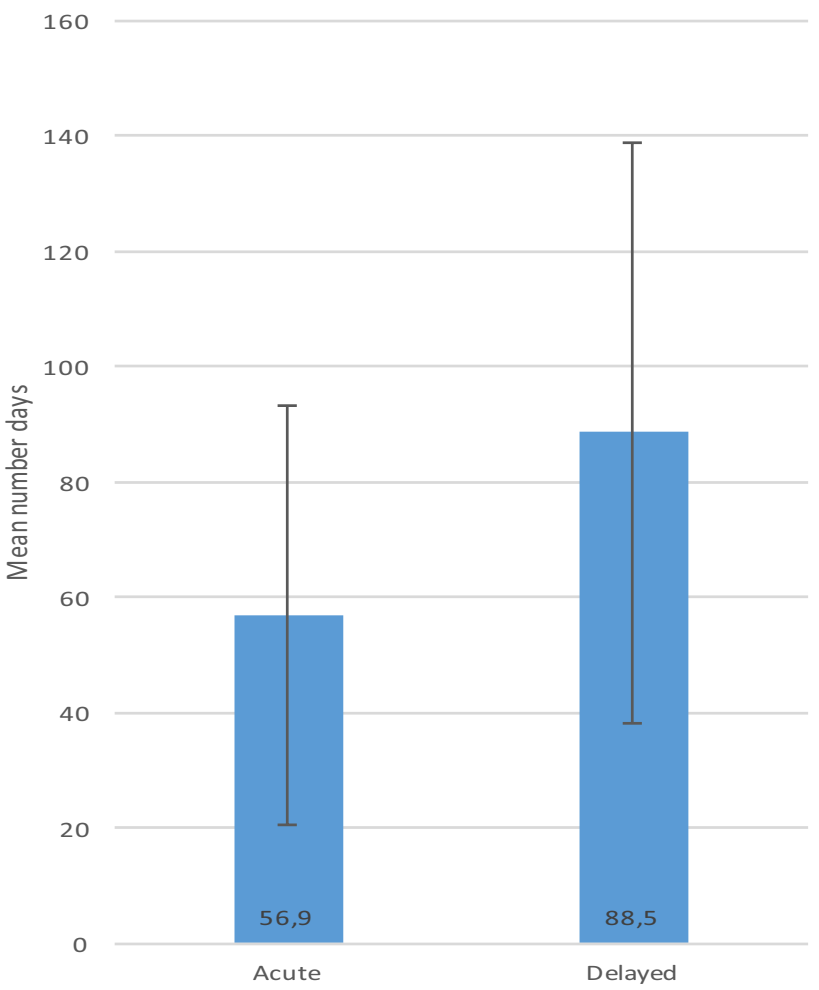

Fig. 2 Total amount sick-leave days
Postoperative sick-leave days

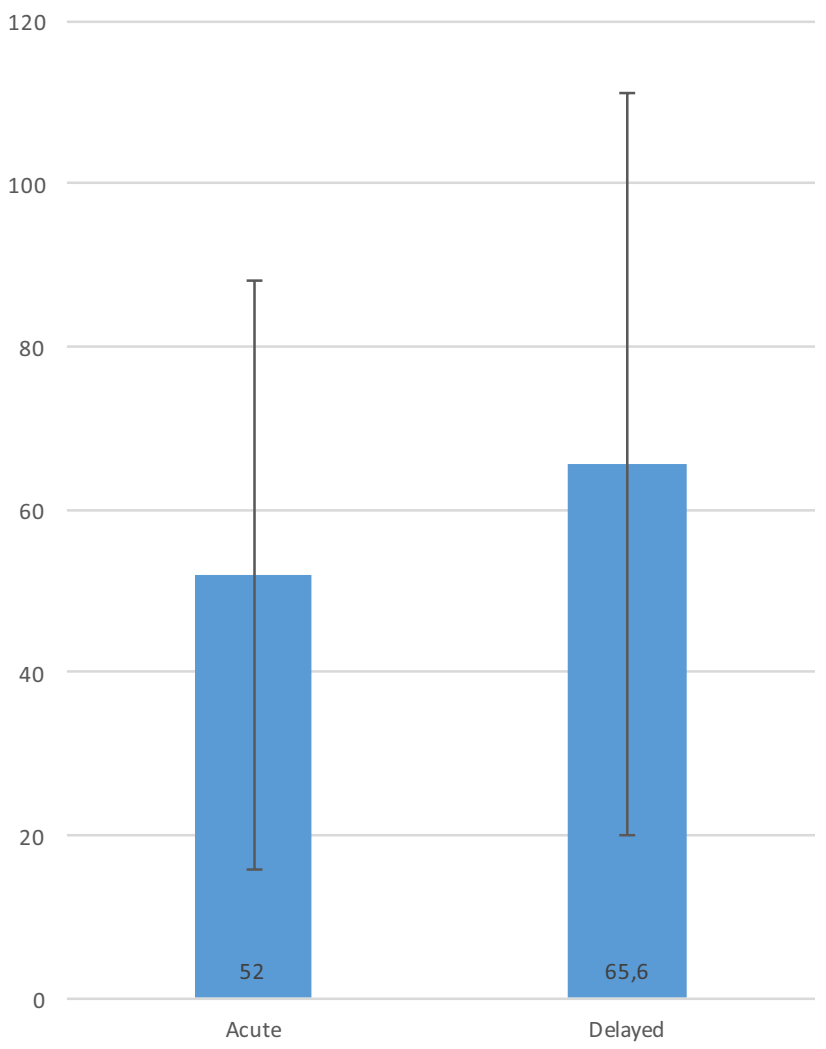

Fig. 3 Postoperative sick-leave days

On average the acute group also had fewer days for recovery after surgery, but not statistically different $(M=52.0$ $\mathrm{SD}=36.1)$ compared to the delayed group $(M=65.6$, $\mathrm{SD}=45.5), p=$ n.s.(0.25) (Fig. 3).

\section{Patient-reported outcome}

After the injury, the acute group was less affected in the KOOS subscales 'pain' and 'quality of life'. After 12 months, the KOOS were similar in the groups (Fig. 4), with significant changes after injury to 12 months, but no significant difference between the groups at any time-point. Both groups had improved Tegner and Lysholm scores from inclusion to the 12-month follow-up (Table 3).

The overall objective, IKDC as well as manual laxity measurements using pivot shift and Rolimeter did not demonstrate any statistically significant differences (Table 3).

Similar ROM between the groups was found at 12 months measured at the hospital unit (Table 3).

No differences were found between the groups in the oneleg hop test or thigh muscle atrophy. At 12 months after reconstruction there were more patients cleared to return to sports in the acute group, however there was no statistical difference. 


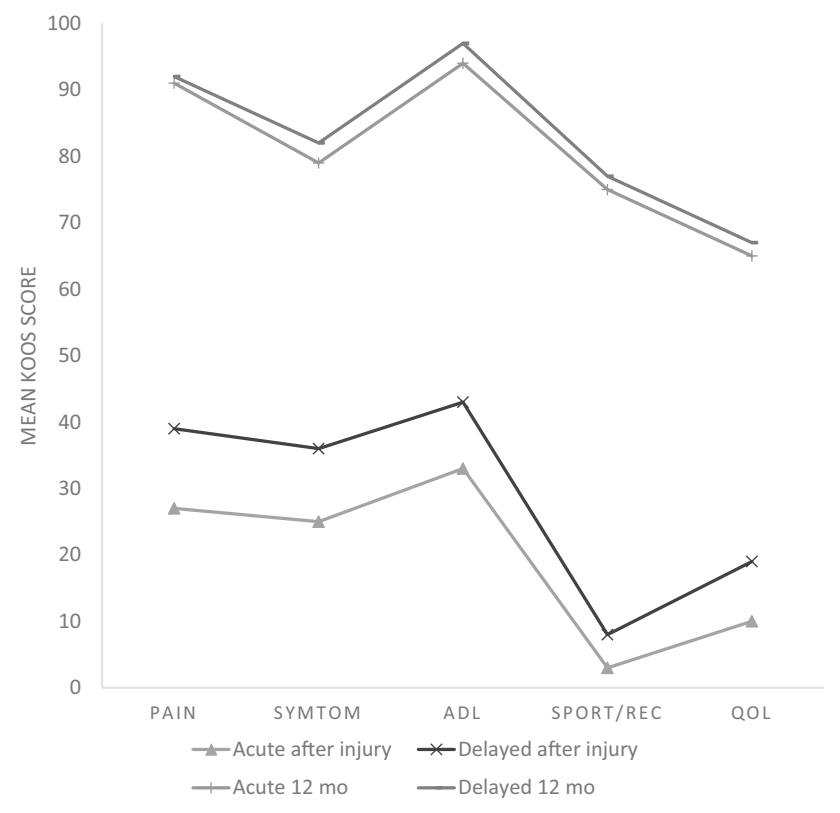

Fig. 4 Mean KOOS scores with significant changes after injury to 12 months, but no significant difference between the groups at any time-point

The muscle strength peak torque in percent of uninjured limb was significant higher for the acute group (Biodex ${ }^{\circledR}$ test).

\section{Discussion}

The major finding of this study was that a reduction in indirect costs (as defined by lost wages and sick-leave days) was seen in the acute ACLR group. These results support the notion that early ACL reconstruction could be more cost-effective than delayed surgical treatment strategies as reported in several studies [19, 20, 26, 27].

In both groups, there was a significant improvement in self-reported quality of life after the injury to 1 year after surgery. There was no difference between the groups with respect to the IKDC score [23], with $83 \%$ of the participants in the acute group scoring either A or B compared to $80 \%$ in the delayed. There were no significant differences in measurements of knee laxity and knee ROM, suggesting that the benefits of acute surgery are primarily seen in the early phase after the surgery and that good results can be achieved after 1 year regardless of timing. There was no significant muscle atrophy of the thigh muscles compared to the contralateral leg in either group at the 12 months follow-up. There was however a significant difference in knee flexion strength, with an advantage for acute surgery, indicating that there is a potential for faster muscle recovery with acute surgery.
The findings in this study are supporting the conclusion of Herbst et al. that ACLR within $48 \mathrm{~h}$ is preferable in highly active patients to avoid unnecessary delay for return to full activity [9]. Our findings are also consistent with those of Maher et al. who found a greater improvement of QALY with ACLR compared to structured rehabilitation alone and concluded that it is the unstable knee that costs the society due to the loss of wages, productivity and disability.

In contrast to the results in this study, Frobell et al. found a lesser QALY improvement after ACLR, and therefore found ACLR to be less cost-effective. They concluded that early reconstruction was not superior to initial nonsurgical treatment with optional delayed reconstruction and stated that early reconstruction did not have any economic benefits $[21,28]$. A consideration regarding this conclusion is the number of patients in the study that underwent reconstruction in the optional delayed group. At the 5-year followup $51 \%$ had undergone delayed reconstruction and these patients required significantly more meniscus procedures compared to those who underwent early reconstruction [29]. The study by Frobell et al. also excluded sick-leave costs that did not extend over 14 days.

The differences in costs between the two strategies are affected by several variables and can be measured in different ways. As such, using sick days as a proxy for socioeconomic costs has its limitations and may not completely reflect socioeconomic costs. However, this study supports our assertion that a change in the timing of ACL surgery, when indicated, could reduce the socioeconomic costs to society resulting from ACL injury.

It is not always obvious which patients should undergo early reconstruction and which patients will achieve a good outcome with conservative treatment. A patient with high functional demands and risk for giving way, who is not prepared to adjust activities and has an unstable knee immediately post-injury, is generally better treated with acute ACLR. A low-demand patient with below average risk of symptomatic instability can undergo rehabilitation first and undergo delayed ACLR should instability arise. The challenge is often determining which treatment is optimal in patients who do not neatly fit into these two groups, balancing the risks of unnecessary surgery for those who could successfully be managed conservatively against the risks and costs of treatment delay for those who go on to require surgery.

The major strength of this study is the prospective, randomized design with four experienced surgeons performing all of the ACL reconstructions with the same surgical technique. Furthermore, one centre with the same postoperative rehabilitation protocol was used in both groups. Another strength is that the study population was selected from a highly motivated patient category with an activity level that justifies reconstruction and a strict definition of the time 
Table 3 Patient-reported outcomes, knee laxity measures and strength at 12 months
Acute ACL reconstruction Delayed ACL reconstruction $p$ value $(n=31-34)$ $(n=32-34)$

\begin{tabular}{|c|c|c|c|}
\hline \multicolumn{4}{|l|}{ Patient-reported outcomes at 12 months } \\
\hline \multicolumn{4}{|l|}{ Lysholm mean (SD) ${ }^{\mathrm{a}}$} \\
\hline Inclusion & $32(21.5)$ & $43(26.2)$ & n.s \\
\hline 6 months & $76(16.2)$ & $79(15.2)$ & n.s \\
\hline 12 months & $87(18)$ & $88(17)$ & n.s \\
\hline \multicolumn{4}{|l|}{ Tegner median (range) ${ }^{\mathrm{b}}$} \\
\hline Before injury & $8(6-10)$ & $9(5-10)$ & n.s \\
\hline At inclusion & $0(0-6)^{\mathrm{c}}$ & $0(0)$ & 0.001 \\
\hline 6 months & $4(1-9)$ & $4(0-9)$ & n.s \\
\hline 12 months & $6(1-9)$ & $6(0-9)$ & n.s \\
\hline \multicolumn{4}{|l|}{ Instrumented knee laxity } \\
\hline Rolimeter mean mm (SD) & $2.0(1.4)$ & $1.9(1.2)$ & n.s \\
\hline \multicolumn{4}{|l|}{ Mean degrees (SD) w ref CL limb } \\
\hline Extension deficit & $2(2.1)$ & $3(3.3)$ & n.s \\
\hline Flexion deficit & $1.8(2.2)$ & $3.2(3.4)$ & n.s \\
\hline $\begin{array}{l}\text { No }(\%) \text { normal } \\
\text { Pivot shift test }{ }^{\mathrm{d}}\end{array}$ & $30(94)$ & $29(88)$ & n.s \\
\hline \multicolumn{4}{|l|}{ IKDC Score $n(\%)^{\mathrm{e}}$} \\
\hline \multicolumn{4}{|l|}{6 months } \\
\hline $\mathrm{AB}$ & $27(82)$ & $24(71)$ & n.s \\
\hline $\mathrm{CD}$ & $6(18)$ & $10(29)$ & \\
\hline \multicolumn{4}{|l|}{12 months } \\
\hline $\mathrm{AB}$ & $26(84)$ & $27(80)$ & n.s \\
\hline $\mathrm{CD}$ & $5(16)$ & $7(20)$ & \\
\hline \multicolumn{4}{|l|}{ Functional strength } \\
\hline $\begin{array}{l}\text { Thigh deficit circ. } 10 \mathrm{~cm} \text { above patella diff } \\
\text { in } \mathrm{cm} \text { ref } \mathrm{CL}\end{array}$ & 0.8 & 1.24 & n.s \\
\hline \multicolumn{4}{|l|}{ One-leg hop $n(\%)^{\mathrm{f}}$} \\
\hline$>90$ & $23(74)$ & $21(63)$ & n.s \\
\hline $76-89$ & $6(19)$ & $8(24)$ & \\
\hline $50-75$ & $1(3)$ & $2(6)$ & \\
\hline$<50$ & $1(3)$ & $2(6)$ & \\
\hline \multicolumn{4}{|l|}{ Muscle strength Biodex ${ }^{\circledR}$} \\
\hline \multicolumn{4}{|l|}{ Peak torque $\%$ ref to CL limb } \\
\hline \multicolumn{4}{|l|}{ Ext. isokinetic } \\
\hline $60 \% \mathrm{~s}$ & 90.2 & 82.9 & 0.08 \\
\hline $180 \% \mathrm{~s}$ & 91.7 & 86.5 & n.s \\
\hline \multicolumn{4}{|l|}{ Flex. isokinetic } \\
\hline $60 \% \mathrm{~s}$ & 95.6 & 88.3 & 0.005 \\
\hline $180^{\circ} / \mathrm{s}$ & 96.7 & 90.2 & 0.01 \\
\hline
\end{tabular}

$A C L$ anterior cruciate ligament, $C L$ uninjured contralateral limb

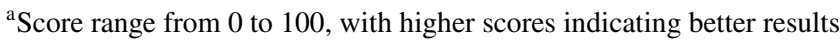

${ }^{\mathrm{b}}$ Assesses activity level with specific emphasis on knee; scores range from 1 (least strenuous activity) to 10 (high knee demanding activity on professional sports level) 15

${ }^{\mathrm{c}}$ One patient answered 6 at inclusion, misunderstood guidelines

${ }^{\mathrm{d}}$ Assesses rotational stability of knee at rest result range from 0 (normal stability) to 3 (severely increased instability)

${ }^{\mathrm{e}}$ Assesses knee function, $\mathrm{AB}$ normal or near normal, $\mathrm{CD}$ abnormal

${ }^{\mathrm{f}}$ Result indicates if the patient is ready to return to play. To pass, the involved leg must measure at least $90 \%$ of the distance compared to the uninvolved leg

${ }^{g}$ Comparison of extensor and flexor torque deficits collected for isometric Biodex, displayed as mean percentage with reference uninjured CL set at 100 
points for surgical intervention based on the time from injury to surgery in the acute group was used. Furthermore, the loss to follow-up rate was negligible $(n=1)$ regarding data on sick-leave.

The results of this study should however be interpreted in light of some limitations. Firstly, the pre-study power analysis was made to detect differences in range of motion and not days of sick leave. However, the differences between the groups are large which increases the likelihood of the results being true even with a post hoc power analysis of $70 \%$. Secondly, despite there being several other factors that affect the total cost of ACLR, we have only assessed the number of sick-leave days as a proxy for socioeconomic costs. We also did not have a nonoperative counterpart for comparison and our study is only focused on individuals with at least a moderate activity level.

As for clinical relevance, this study demonstrates that acute ACLR can be performed safely in highly active patients and ensure an earlier return to work and full activity.

\section{Conclusion}

Acute and delayed ACLR provided comparable clinical outcomes after 12 months. Acute reconstruction resulted in less sick-leave days and as such fewer indirect costs to the individual and society. These findings suggest that if patients requiring ACLR can be identified early and ACLR can be performed in the acute phase, socioeconomic costs can potentially be reduced by minimizing time off work.

Funding No funding has been received for this study.

\section{Compliance with ethical standards}

Conflict of interest The authors have no conflicts of interest.

Ethical approval The study was approved by the regional ethics committee at the Karolinska Institute, Stockholm Sweden (reference no. 2006/404-31/3/2008/1541-32).

Informed consent Informed consent was obtained by each patient.

Open Access This article is distributed under the terms of the Creative Commons Attribution 4.0 International License (http://creativeco mmons.org/licenses/by/4.0/), which permits unrestricted use, distribution, and reproduction in any medium, provided you give appropriate credit to the original author(s) and the source, provide a link to the Creative Commons license, and indicate if changes were made.

\section{References}

1. Spindler KP, Wright RW (2008) Clinical practice. Anterior cruciate ligament tear. N Engl J Med 359:2135-2142

2. Swedish Knee Ligament Register (2018): “The Swedish National ACL Register". https://aclregister.nu/media/uploads/Annual\%20 reports/rapport2016en.pdf. Accessed 18 Dec 2018

3. Frobell RB, Roos HP, Roos EM, Roemer FW, Ranstam J, Lohmander LS (2013) Treatment for acute anterior cruciate ligament tear: five year outcome of randomised trial. BMJ 346:f232

4. Grindem H, Eitzen I, Engebretsen L, Snyder-Mackler L, Risberg MA (2014) Nonsurgical or surgical treatment of ACL injuries: knee function, sports participation, and knee reinjury: the Delaware-Oslo ACL Cohort Study. J Bone Jt Surg Am 96:1233-1241

5. Grindem H, Wellsandt E, Failla M, Snyder-Mackler L, Risberg MA (2018) Anterior cruciate ligament injury-who succeeds without reconstructive surgery? The Delaware-Oslo ACL Cohort Study. Orthop J Sports Med 6:2325967118774255

6. Wittenberg RH, Oxfort HU, Plafki C (1998) A comparison of conservative and delayed surgical treatment of anterior cruciate ligament ruptures. A matched pair analysis. Int Orthop 22:145-148

7. Bottoni CR, Liddell TR, Trainor TJ, Freccero DM, Lindell KK (2008) Postoperative range of motion following anterior cruciate ligament reconstruction using autograft hamstrings: a prospective, randomized clinical trial of early versus delayed reconstructions. Am J Sports Med 36:656-662

8. Eriksson K, von Essen C, Jönhagen S, Barenius B (2018) No risk of arthrofibrosis after acute anterior cruciate ligament reconstruction. Knee Surg Sports Traumatol Arthrosc 26:2875-2882

9. Herbst E, Hoser C, Gföller P, Hepperger C, Abermann E, Neumayer K et al (2017) Impact of surgical timing on the outcome of anterior cruciate ligament reconstruction. Knee Surg Sports Traumatol Arthrosc 25:569-577

10. Smith TO, Davies L, Hing CB (2010) Early versus delayed surgery for anterior cruciate ligament reconstruction: a systematic review and meta-analysis. Knee Surg Sports Traumatol Arthrosc 18:304-311

11. Shelbourne KD, Johnson GE (1994) Outpatient surgical management of arthrofibrosis after anterior cruciate ligament surgery. Am J Sports Med 22:192-197

12. Shelbourne KD, Patel DV (1995) Timing of surgery in anterior cruciate ligament-injured knees. Knee Surg Sports Traumatol Arthrose 3:148-156

13. Shelbourne KD, Wilckens JH, Mollabashy A, DeCarlo M (1991) Arthrofibrosis in acute anterior cruciate ligament reconstruction. The effect of timing of reconstruction and rehabilitation. Am J Sports Med 19:332-336

14. Brambilla L, Pulici L, Carimati G, Quaglia A, Prospero E, Bait C et al (2015) Prevalence of associated lesions in anterior cruciate ligament reconstruction: correlation with surgical timing and with patient age, sex, and body mass index. Am J Sports Med 43:2966-2973

15. Church S, Keating JF (2005) Reconstruction of the anterior cruciate ligament: timing of surgery and the incidence of meniscal tears and degenerative change. J Bone Jt Surg Br 87:1639-1642

16. Granan LP, Bahr R, Lie SA, Engebretsen L (2009) Timing of anterior cruciate ligament reconstructive surgery and risk of cartilage lesions and meniscal tears: a cohort study based on the Norwegian National Knee Ligament Registry. Am J Sports Med 37:955-961

17. Lohmander LS, Englund PM, Dahl LL, Roos EM (2007) The long-term consequence of anterior cruciate ligament and meniscus injuries: osteoarthritis. Am J Sports Med 35:1756-1769

18. Sanders TL, Kremers HM, Bryan AJ, Fruth KM, Larson DR, Pareek A et al (2016) Is anterior cruciate ligament reconstruction effective in preventing secondary meniscal tears and 
osteoarthritis? Am J Sports Med. https://doi.org/10.1177/03635 46516634325

19. Mather RC 3rd, Hettrich CM, Dunn WR, Cole BJ, Bach BR Jr, Huston LJ et al (2014) Cost-effectiveness analysis of early reconstruction versus rehabilitation and delayed reconstruction for anterior cruciate ligament tears. Am J Sports Med 42:1583-1591

20. Saltzman BM, Cvetanovich GL, Nwachukwu BU, Mall NA, BushJoseph CA, Bach BR Jr (2016) Economic analyses in anterior cruciate ligament reconstruction: a qualitative and systematic review. Am J Sports Med 44:1329-1335

21. Kiadaliri AA, Englund M, Lohmander LS, Carlsson KS, Frobell RB (2016) No economic benefit of early knee reconstruction over optional delayed reconstruction for ACL tears: registry enriched randomised controlled trial data. Br J Sports Med 50:558-563

22. Tegner Y, Lysholm J (1985) Rating systems in the evaluation of knee ligament injuries. Clin Orthop Relat Res 198:43-49

23. Hefti F, Müller W, Jakob RP, Stäubli HU (1993) Evaluation of knee ligament injuries with the IKDC form. Knee Surg Sports Traumatol Arthrosc 1:226-234

24. Roos EM, Roos HP, Ekdahl C, Lohmander LS (1998) Knee injury and Osteoarthritis Outcome Score (KOOS) — validation of a Swedish version. Scand J Med Sci Sports 8:439-448

25. Taylor NA, Sanders RH, Howick EI, Stanley SN (1991) Static and dynamic assessment of the Biodex dynamometer. Eur J Appl Physiol Occup Physiol 62:180-188
26. Lubowitz JH, Appleby D (2011) Cost-effectiveness analysis of the most common orthopaedic surgery procedures: knee arthroscopy and knee anterior cruciate ligament reconstruction. Arthroscopy 27:1317-1322

27. Mather RC, Koenig L, Kocher MS, Dall TM, Gallo P, Scott DJ et al (2013) Societal and economic impact of anterior cruciate ligament tears. J Bone Jt Surg Am 95:1751-1759

28. Frobell RB, Roos EM, Roos HP, Ranstam J, Lohmander LS (2010) A randomized trial of treatment for acute anterior cruciate ligament tears. N Engl J Med 363:331-342

29. Frobell RB, Roos HP, Roos EM, Roemer FW, Ranstam J, Lohmander LS (2013) Republished research: treatment for acute anterior cruciate ligament tear: five year outcome of randomised trial. Br J Sports Med 47:373

Publisher's Note Springer Nature remains neutral with regard to jurisdictional claims in published maps and institutional affiliations. 